\title{
Developing a Conceptual Definition of the Successfully Transitioned Newly Licensed Registered Nurse (NLRN)
}

\author{
Joset Brown* and Jill Nocella
}

*Correspondence: brownj127@wpunj.edu

\begin{abstract}
Background: The transition from student to practicing nurse has been described as tumultuous for the newly licensed registered nurse (NLRN). New nurses face challenges with adapting to new and unfamiliar roles and environments while also needing to provide safe nursing care. The consequences of the trepidations are costly and may result in low job satisfaction, high job turnover rates, and poor patient outcomes.

Methods: Concept development was conducted adhering to the three-step Schwartz-Barcott and Kim's hybrid model. Twenty-two articles that met inclusion criteria from PubMed, MEDLINE, and Cumulative Index to Nursing and Allied Health Literature (CINAHL) were reviewed and analyzed. Second, semistructured interviews with 17 registered nurses to explore their perceptions of the attributes of a successfully transitioned NLRN were conducted, followed by inductive content analysis. Finally, a comprehensive definition of the successfully transitioned NLRN was developed.

Results: The literature search failed to reveal a definition of the successfully transitioned NLRN. Evaluation of existing tools from the literature review yielded no clear conclusions or attributes of transitional success. Based on this finding and supported by the semi-structured interviews, a definition of the successfully transitioned NLRN is posited.

Conclusion: A precise definition of a successfully transitioned nurse provides a consistent benchmark to measure progress toward independent practice. This definition supports the development of a psychometrically validated instrument to operationalize and measure attributes. This is a crucial step to determine the need for and the amount of support required as the NLRN continues to evolve professionally.

Keywords: Successful transition, New nurse, Newly licensed nurse, Transition to practice
\end{abstract}

\section{Introduction}

The transition from the role of student to being in the practice role has been described as tumultuous for the newly licensed registered nurse (NLRN) related to challenges with adapting to new and unfamiliar roles and environments while also needing to provide safe nursing care [1-4]. The experiences of the NLRN's transition from academia to clinical practice has been described in the literature as a "reality shock", [5] "culture shock", [6] and "transitional shock" [7] .

The challenges these nurses face include the dynamic and changing nature of the healthcare environment: staffing ratios, patients with increasing acuities, a more complex health care environment, and reduced clinical exposure due to reduced clinical placement sites and a perceived incongruence between expectation and reality [8-10]. Though the difficulties experienced during this phase are varied, the consequences of the trepidations faced during this phase are costly, result in low job satisfaction, high job turnover rates, and poor patient outcomes [11-16].

To address this concern, efforts have emerged to assist the nurse through this demanding phase, including transitional support programs and mentoring $[11,17]$. With no standard nomenclature, these support programs have been labeled as "nurse residency programs, nurse internship programs, or transition to practice programs," which serve to provide support as the NLRN transitions to independent practice $[18,19]$. Though helpful, these programs are inconsistent and vary in duration, 
content, and structure. The wide variation and lack of consistency may result in a disparity with uniformity in competence and performance levels of NLRNs who then enter practice independently.

Despite its pervasiveness in the literature, the successful transition of the NLRN remains a largely ill-defined concept. Much of the literature describes the concept of practice readiness, transition shock, and the theory-practice gap; however, none of these terms relate to the completion of a period resulting in successfully transitioning to independent clinical practice. The absence of a clear definition likely contributes to the lack of identification of determinants of achievement of this level of success and a lack of a survey instrument to measure these attributes specifically. The development of a definition can first provide some fundamental principles for evaluating the nurse as they progress through the transitional process, and second, identify the attributes to measure their success. Therefore, this study's purposes were to (1) analyze and explore the attributes of a successful transition and (2) develop a conceptual definition of the successfully transitioned NLRN.

\section{Methods}

\section{Study Design}

The concept of the successfully transitioned NLRN was analyzed using the hybrid model developed by Schwartz-Barcott and Kim [20] which includes three phases: theoretical, fieldwork, and the final analysis phase. This three-step hybrid model was deemed the most appropriate for the development of this concept due to its inclusion of nurses' perspectives, which enriches the limited research literature [21]. The theoretical phase is initiated with the selection of a concept and examination of the available literature resulting in the formulation of a working definition. In the fieldwork phase, the concept is analyzed and clarified using qualitative methods. The final analysis phase constitutes a conceptual analysis of the findings obtained from the previous two phases to identify the concept's attributes, further clarifying its meaning and providing a definition [20].

\section{Data Collection}

\section{The theoretical phase}

The databases of PubMed, MEDLINE, and Cumulative Index to Nursing and Allied Health Literature (CINAHL), were searched with a combination of keywords (graduate nurse or newly licensed nurse and successful transition to practice or transition to practice). Primary studies ( $n=22)$ that met inclusion criteria of defining attributes for the successful transition of NLRNs during the years 2010 to 2020 in the United States, and instruments measuring attributes of transition $(n=7)$ referenced in these studies were retained and appraised. Quality of the review was assured by adhering to Preferred Reporting Items for Systematic Reviews and MetaAnalysis (PRISMA) guidelines (Figure 1) and having two nurse researchers screen and extract all data. Duplicate records were removed, then abstracts were reviewed for relatedness to the concept, then finally, full texts were reviewed for eligibility for inclusion in the study. Studies that were conducted outside of North America, program evaluations, and reported perceptions and attitudes of preceptors, educators, and nurse managers were excluded. The articles were analyzed systematically to determine the working definition and attributes related to the concept under investigation.

\section{The fieldwork phase}

In this phase, registered nurses were interviewed to explore their perceptions of the successfully transitioned NLRN and to validate the findings of the theoretical phase. Snowball sampling was used as a cost-effective method to gain access to and recruit participants from diverse healthcare settings and different geographic locations at an accelerated pace. Participants recruited for this study were licensed and currently practicing in a healthcare environment, with a minimum of 12 months experience as a registered nurse. There were no exclusions based on age, gender, ethnicity, educational preparation, employment agency, or geographic location. In addition, nurse administrators were also recruited to share their perceptions. Initial potential participants were identified from an alumni mailer list provided by the department of nursing at William Paterson University. The final sample size was 17 participants $(\mathrm{N}=17)$, representing a total of seven hospitals within the United States with 12 registered nurses and five nurse administrators (nurse managers, nurse educators, and a staff development professional). The difference in numbers of the group was due to participant availability.

To ensure the data's accuracy, individual interviews using zoom technology continued until data saturation was achieved and no new data emerged. Interviews were eight to 20 minutes in duration (mean 15 minutes) and were audio-recorded with the participants' permission, then transcribed verbatim. To assure anonymity, the data was deidentified, and participant names were replaced with pseudonyms.

The interview guiding questions at this phase are presented below:

For the registered nurse (In a non-leadership role)

- What attributes do you believe a successfully transitioned nurse possesses?

- Do you consider yourself successfully transitioned in your practice?

- How long before you considered yourself to be successfully transitioned?

- What factors do you believe support the successful transition of the NLRN?

- What factors do you believe detract from the successful transition of the NLRN?

- How would you define a successfully transitioned NLRN?

For the registered nurse (In a leadership role)

- What attributes (knowledge, skills, or attitudes) do you believe a successfully transitioned nurse should possess?

- In your experience, how long does it take for a NLRN to 


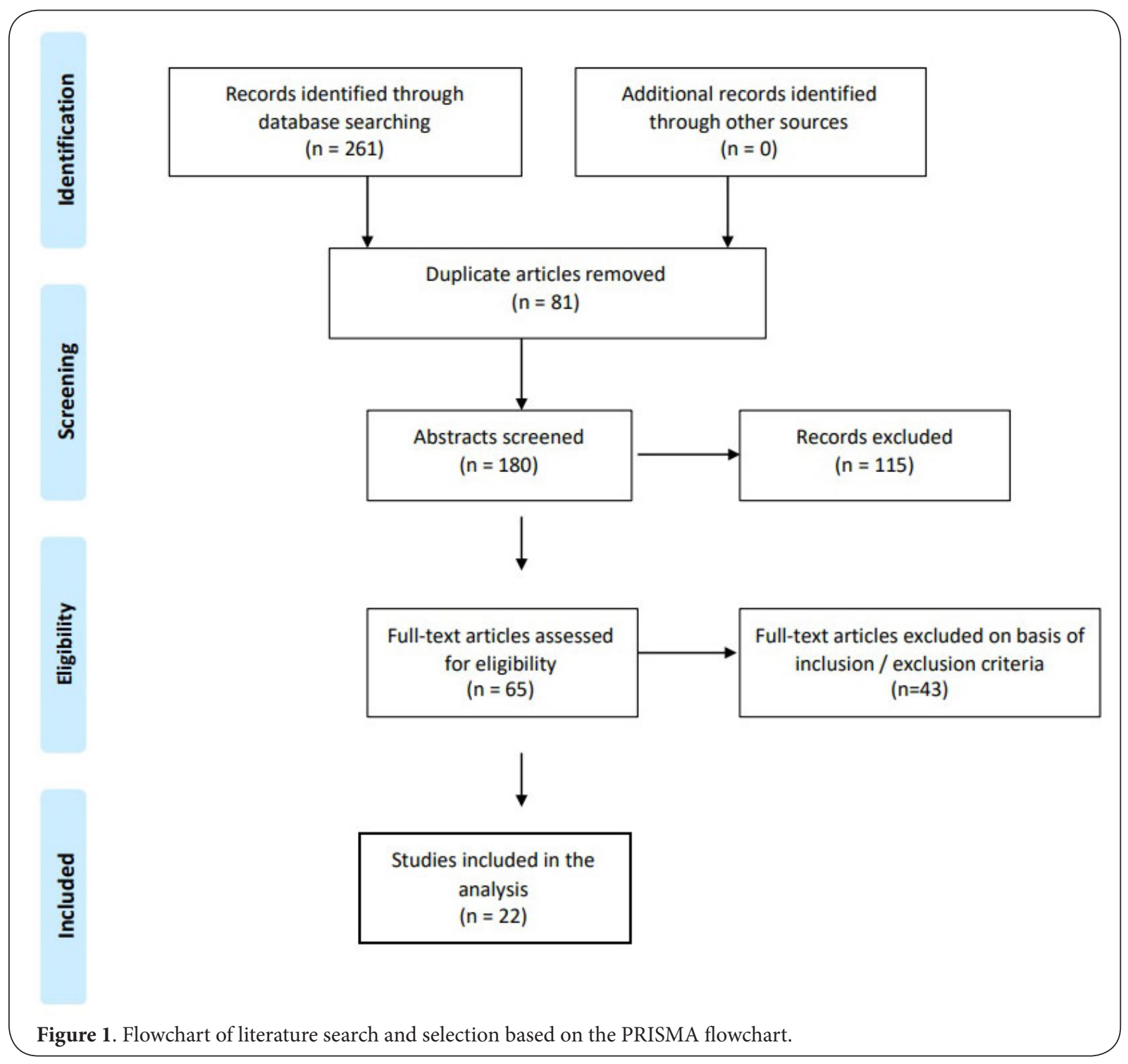

attain these qualities?

- What factors do you believe support the successful transition of a NLRN?

- What factors do you believe detract from the successful transition of the NLRN?

- How would you define a successfully transitioned NLRN?

To increase the dependability of the findings from the interviews, data were transcribed by one of the researchers immediately following each interview. Field notes, noting the context of the interviews, were also collected. The fieldwork phase lasted from August to December 2020.

\section{Final analytical phase}

In the final analytical phase, findings from the theoretical and the fieldwork phases were compared to produce a refined and comprehensive definition supported by research and the perceptions of the participants [20]. The concept of the success- fully transitioned NLRN was defined reliant on the emerging themes and attributes.

\section{Data Analysis}

In the theoretical phase, conventional content analysis of the text was conducted, and units were coded and analyzed into three categories: antecedents, attributes, and consequence. In the second phase, data analysis was achieved through an iterative and inductive process, adhering to Moustaka's modified version of the Stevick-Coalazzi-Keen's method of data analysis as summarized and presented by Creswell [22,23]. The researchers employed the following procedure: (a) immersion in the data by listening to the audio-recorded interviews several times, transcribing verbatim, and reviewing and rereading all the interview transcripts, to explore and ascertain possible meanings of the data; (b) evaluating each statement based on its own value; (c) methodically categorizing significant state- 
Brown et al, Journal of Nursing 2021,

ments into discrete codes; and (d) organizing and revising the codes utilizing thematic analysis which resulted in 3 themes.

A team-based approach to analyze the data was used to confirm the credibility of the study. The transcripts were reviewed and revised by the research team and coded. Peer review and member checking helped ensure trustworthiness [24]. Two expert nursing educators who were not involved in the study reviewed and provided feedback on the emerging definition. Upon receipt of the feedback, the definition was refined to incorporate their comments. For example, expert one determined the use of "basic" to describe proficiency did not accurately capture the level of competence expected from a NLRN who has successfully transitioned, but rather one who has just entered the role. Based on this feedback, the term was eliminated and replaced with "functional proficiency". Following this process, each participant was emailed the definition to determine if it accurately reflected their perceptions and experiences with the concept. Thirteen of the 17 participants responded and approved of the definition with no further amendments. All documents were securely stored, accessible only to the two researchers to enable further investigation.

\section{Ethical Considerations}

Participant recruitment commenced following institutional review board approval.

Informed consent was obtained prior to the interview process. All participants were reminded of the aims of the study prior to the start of each interview. Participation was voluntary, and no compensation was offered.

\section{Results}

While a great deal is known about the active transitioning process of the NLRNs within their first years of clinical practice, the search failed to reveal a precise and comprehensive definition of the successfully transitioned NLRN or a tool to measure their attributes. According to the findings, a successful transition is often used interchangeably with 'practice readiness' or "job readiness' and is said to be the result of a 'theory-practice gap' or 'gap' between theory and practice [25]. Despite the identification and concern regarding the deficiencies experienced by novice nurses, there is a lack of a definition of "readiness" as a concept and suggests continued refinement and exploration as healthcare environments increase in complexity [26].

\section{Theoretical phase}

\section{Characteristics and definition of the concept}

For the purposes of this study, the NLRN is defined as a nurse within two years of graduation [27]. According to the MerriamWebster dictionary [28], transition implies a state of change or evolution from one form or state to another. In nursing, role transition refers to a period during which a nurse experiences adjustment and socialization to a new role and or work environment [29]. This transitional period is a time of professional growth, which also involves learning new roles and the culture of the new environment. Kramer [5] used the term 'reality shock' to describe the hardships faced when graduate nurses experience clinical practice realities. The experiences of the NLRN have also been referred to as "culture shock" [6] and "Transition shock" [7,30].

Benner's Novice to Expert theory describes the nurse's evolution or transition through a fivephase period of being a novice culminating in being an expert [31]. These stages indicate a progression in the acquisition of knowledge, confidence, and competence to gain a level of expertise. Duchscher's stages of transitions theory [7] provided a model of transition, identifying the process of "becoming" a time of development when the nurse progresses through the stages of knowing, doing, and being, evolving through the first twelve months of practice. However, she adds the NLRN faces what she describes as transition shock on entry to practice through most acutely felt during the first three to four months of transitioning from student to the practice role [7].

Practice readiness has been defined several different ways within the literature [32]. One way is having confidence in clinical skills and the ability to readily assimilate and apply new knowledge [8,33]. A concept analysis by Mirza et al., [34] found that practice readiness revolved around the concept of safe care, performance confidence, and having both the cognitive and clinical capability to perform a nurse's role. The nurse's performance is often described based on competence. Competence refers to the nurse's ability to use knowledge in a particular context or situation [34].

\section{Attributes}

The attributes that emerged from the existing studies reviewed in the theoretical phase can be categorized as follows:

Cognitive development (development of critical thinking, clinical reasoning, and clinical judgment).

Clinical competence (Skill mastery, prioritization of care, delegation, time management).

Professional development (professional behaviors developed through mentoring relationships and supportive environments, resulting in socialization to their professional roles).

Successfully transitioned nurses adjust to their new roles and expectations and are proficient in problem-solving and executing basic nursing skills and functions $[19,10]$. Nurses deemed competent in their practice excel in time management and skill mastery; $[4,16,33]$ prioritization, communication, professionalism, and teamwork; $[16,19,35]$ and can collaborate effectively as part of a team [36]. Communication is paramount to collaboration among the multidisciplinary team, which is essential to promote patient safety and continuity of care [36]. The development of critical thinking is crucial for NLRNs to function independently, provide safe care, and engage in effective clinical decision-making [35]. Additionally, nurses demonstrate competence in their practice through an ability to skillfully/effectively adjust their plan of care based on changes in patient status [16]. 
While it is important for nurses to be competent in their practice, another attribute of successfully transitioned nurses is integrating their knowledge appropriately in practice and making sound clinical decisions [37]. Novice nurses facing difficulties entering practice demonstrate difficulties in key areas such as critical thinking, organization, communication, and leadership [35].

\section{Antecedents}

The antecedents of transitional success in the related literature fit into two categories:

Acquisition of skill competence (Gained through experience and exposure).

Supportive organizational culture (Through supportive professional environments - mentoring relationships, socialization to the practice role, structured transitional support programs).

Most of the researchers noted the importance of the nurse's confidence and competence in skill mastery and the ability to integrate knowledge fluidly in the clinical area as antecedents for a successful transition to practice. According to Benner [31], nurses develop professional competence and expertise in practice through experiential learning, suggesting a benefit of development through experience and exposure over time [37].

Work-related environmental experiences were also found to significantly influence the transition experience [33]. Professional and emotional support and a period of orientation in the post-hire period are helpful to familiarize the novice nurse to their new role and organization. Nurse's transition into their roles more successfully when they are provided with consistent supportive, experienced, and resourceful preceptors or clinical coaches to socialize them to their professional roles and the organization $[4,19,38,39,13,26]$. These supportive relationships assist in building confidence $[15,36,40]$, reducing anxiety $[9,39]$, and promoting job satisfaction $[19,38]$.

The assimilation process into the work environment provides support through and past the orientation period $[4,19,7,10]$. An environment of this nature allows for comprehensive support for the NLRN to develop their professional identity and practice, socialize into their new environment and roles, and promote confidence and competence in their performance and decision-making skills $[7,10]$. Participation in transitional support programs reduced attrition rates and increased proficiency, confidence and performance $[9, \mathbf{4 0}]$. In contrast, lack of support, incivility, and lack of clarity of role expectations in the clinical environment increases anxiety and hinders a successful transition [9].

\section{Consequences}

The demonstrated consequences of a successful transition include:

Job satisfaction (retention / reduced job turnover).

Patient safety and positive healthcare outcomes Professional excellence (Practice readiness (Skill mastery), seamless integration of theory into practice).
Though the term was not clearly defined in the literature, the consequences of a successful transition were very apparent. A successful transition is paramount to provide safe care to obviate sentinel events and negative healthcare outcomes. A successful transition for the new nurse results in autonomy and the development of critical thinking skills [9,35]; attributes necessary for functioning in clinical environments with heightened complexities of patient populations.

A successful transition involves more than task mastery, completion of a period of orientation, or a prescribed transitional support program. A successful transition takes into account the NLRN's ability to integrate knowledge appropriately and demonstrate a functional / basic proficiency and confidence in practical skills such as communication, collaboration, delegation, advocacy, and clinical decision making independently and seamlessly assimilate into their professional roles. Conversely, difficult transitions affect the nurse's ability to make clinical decisions [35], creates feelings of uncertainty, increases anxiety, decreased confidence [38], and increases new-nurse attrition resulting in NLRNs leaving clinical practice [41], often within their first year of employment [15].

Nurses who successfully are transitioned in their practice demonstrate effective communication (written and verbal), leadership, delegation, prioritization, time management, and organization [35]. The outcome of the successful transition of the NLRN is increased job satisfaction with intent to remain in the profession and enhanced patient safety $[37,39,16,41]$. The level of performance anticipated with a successful transition mirrors Benner's competent phase of skill acquisition. According to Benner [41], a competent nurse has completed 2-3 years of practice and has developed the ability/expertise to prioritize and safely and efficiently care for clinically challenging patients.

Given the lack of a definition of the successfully transitioned nurse, no tools were identified that explicitly measured the concept. Seven tools were identified throughout the literature review that have been used to measure graduate nurse satisfaction, experience, critical thinking, and transitional program evaluation.

\section{Working definition}

Based on the review of the literature, a working definition of the concept of the successfully transitioned NLRN was constructed:

A successfully transitioned nurse has completed a period of orientation, and demonstrates confidence in applying their knowledge, competence in skill performance, and is comfortable in their working environment as seen in their communication and collaboration with the patient and healthcare team.

\section{The fieldwork phase}

3. Sample characteristic

Seventeen registered nurses participated in interviews. The demographics of the participants are provided in Table 1. The difference in numbers of the groups was due to participant availability. Nurse administrators identified as nurse managers, 
Brown et al, Journal of Nursing 2021,

Table 1. Demographic data.

\begin{tabular}{ccccccl}
\hline Participant & Gender & $\begin{array}{c}\text { Age } \\
\text { Range }\end{array}$ & Role & $\begin{array}{c}\text { Clinical } \\
\text { Unit }\end{array}$ & $\begin{array}{c}\text { Degree } \\
\text { Earned }\end{array}$ & $\begin{array}{c}\text { Duration of } \\
\text { experience }\end{array}$ \\
\hline $\mathbf{1}$ & Female & $45-50$ & Nurse manager & ER & MSN & 26 years \\
$\mathbf{2}$ & Female & $40-45$ & Clinical coordinator & ICU & MSN & 28 years \\
$\mathbf{3}$ & Male & $25-30$ & Staff nurse & Med surg & BSN & 22 years \\
$\mathbf{4}$ & Female & $20-25$ & Staff nurse & LTAC & Diploma & 4 years \\
$\mathbf{5}$ & Female & $25-30$ & Staff nurse & Med surg & BSN & 6 years \\
$\mathbf{6}$ & Female & $35-40$ & Clinical educator & Med surg & BSN & 18 years \\
$\mathbf{7}$ & Female & $50-55$ & Nurse manager Staff & Med surg & MSN & 7 years \\
$\mathbf{8}$ & Female & $20-25$ & nurse & Dialysis & Diploma & 2 years \\
$\mathbf{9}$ & Female & $35-40$ & Nurse manager & ER & BSN & 5 years \\
$\mathbf{1 0}$ & Female & $40-45$ & Nursing professional & Med surg & MSN & 20 years \\
& & \multicolumn{7}{|c|}{ development specialist } & Nursing faculty & School & PhD & 16 years \\
$\mathbf{1 1}$ & Female & $45-55$ & Staff nurse & Med surg & Diploma & 2.5 years \\
$\mathbf{1 2}$ & Female & $20-25$ & Staff nurse & LTAC & Diploma & 3 years \\
$\mathbf{1 3}$ & Female & $30-35$ & Staff nurse & ER & BSN & 5 years \\
$\mathbf{1 4}$ & Female & $35-40$ & Staff nurse & ICU & BSN & 1.5 years \\
$\mathbf{1 5}$ & Female & $30-35$ & Staff nurse & Med surg & MSN & 6 years \\
$\mathbf{1 6}$ & Female & $35-40$ & Staff nurse & Hospice & BSN & 3 years \\
$\mathbf{1 7}$ & Male & $25-30$ & & & &
\end{tabular}

clinical coordinators, clinical educators, nursing faculty, and a nursing professional development specialist (NPDS).

Semi-structured interviews were conducted to determine the attributes of the successfully transitioned NLRN. The attributes identified by the registered nurses in a non-leadership role were similar to those identified by the nurse administrators. The initial codes were closely analyzed and explored, and three main themes emerged: "knowledge and competency levels" "personal attitudes," and "professional and clinical behaviors. "The themes are presented and supported by verbatim participant comments.

\section{Knowledge and competency levels}

All participants agreed that demonstrating theoretical knowledge and competence in skill performance did not equate to transitional success. A common concern was that many NLRNs have the required knowledge but fail to apply that knowledge appropriately in the clinical area; a nurse manager voiced, "Completing nursing tasks well is not the problem. I am more concerned with their ability to think and apply their knowledge on my unit."

A nurse with 4 years' experience recalled a successful transition for her was not tied to competence in performing nursing skills and completion of tasks but an ability to integrate her knowledge in understanding the "why" and "seeing the big clinical picture" independently. She added that this is the result of "experience and exposure."

In addition, completion of a designated orientation period while assigned to a preceptor does not assure a successful transition. A clinical coordinator opined that many NLRNs complete their orientation period and still were not ready or were unable to work independently. A nurse with 20 months of clinical experience shared, "I completed a six-week orientation and was placed on nights without a preceptor, and I had a challenging time. I wasn't ready, and I made mistakes."

\section{Personal attitudes}

Personal traits identified by the participants included the ability to problem solve, think critically, be assertive, and the ability to advocate for themselves and their patient's safety. In addition, nurse leaders voiced that nurses must also be aware of their strengths and limitations, possess the willingness to seek assistance, and identify the available resources to support their professional growth and progress.

One nurse manager acknowledged that the expectation is not for the NLRN to be experts when they enter practice but that "... they seek knowledge, ...they should not be stagnant but have a desire to learn, and an independence in seeking knowledge... and have an inquiring mind." Another nurse educator stated the nurses who transition with ease in her department were nurses who exhibited a "willingness to learn, accept critique, and use the feedback to improve their performance."

A common thread among all participants was self-awareness. The ability to know and acknowledge their own strengths and limitations, and an ability to identify and address the areas of weakness. A nurse with five years' experience stated, "...as a new 
nurse, you have to have the mentality to speak up and ask for help." Another nurse added, ".......being able to recognize your own limitations and seek out experiences that will help make you into a better practitioner" as a crucial component to ensuring success.

Several participants voiced that NLRNs being assertive in seeking assistance, seeking knowledge, and advocating for their patients was reliant on their competence and confidence in their ability as a nurse. A nurse shared that a NLRN who has successfully transitioned should embody a "confidence in their knowledge, an understanding there is more to learn, and an ability to know where they can get help if they need it." It was a common belief that the NLRN shared responsibility with the employing organization in ensuring their success. This is demonstrated by a desire to learn and independently seek out opportunities to broaden their experience.

\section{Professional and clinical behaviors}

Finally, the nurse must demonstrate professional attitudes, critical thinking ability, and clinical judgment in the clinical environment to indicate the ability to practice independently. A clinical educator described some professional attitudes as "autonomy, collaborating and respecting the multidisciplinary team and an ability to apply their knowledge safely" through skills such as "prioritization and an ability to know when their patients are deteriorating."

A nurse manager stressed the importance of the NLRN being able to prioritize care as a significant determinant in the patient's outcome and a crucial indicator of transitional readiness.

"This is a skill they have to be proficient in to be able to care for their patients independently." A nurse educator added, "They are novice nurses, but they must have a basic understanding of how to prioritize care and have situational awareness; that is key."

Participants articulated that the NLRN's knowledge, skills, and attitudes may not reflect confidence and competence upon entry to practice but should demonstrate an increasing ability to meet these performance expectations.

Communication is identified as a competence to improve patient outcomes and quality of care by the Quality and Safety Education for Nurses (QSEN) and as a national safety goal by the Joint Commission [36]. The NLRN should be cognizant of the importance of communication in facilitating collaboration and delivery of care [16]. There was consensus among both groups that effective communication was an essential attribute of successfully transitioned nurses. A nurse manager opined ... "ineffective communication can result in error and mistakes in patient care.

The nurses added that the NLRN must also show an interest in their own professional growth and be willing to work as part of the team to be successful. According to the registered nurses in a non-leadership role, factors that detracted from a successful transition included "a lack of organization, lack of preparation, incivility in the clinical area, lack of time management, and a lack of support." Similarly, significant factors identified by the nurses in a leadership role that detracted from and negatively impacted transitions were the NLRN's poor time management and difficulty organizing care for a group of patients, and a lack of support on a unit or organizational level.

\section{Final analytical phase}

The results of the fieldwork phase correspond to those of the theoretical phase. Most of the attributes obtained from the theoretical phase were repeated in the fieldwork phase.

According to empirical data, a successful transition is demonstrated by psychomotor, cognitive, and professional development impacted by the clinical environment, organizational support, and personal characteristics of the NLRN. A successful transition is achievable; however, it is an individual evolution from entry to practice to the development of competence and proficiency. The consequence of a successful transition impacts the nurse, patient outcomes, and the employing agency. Entering independent clinical practice without being adequately transitioned results in decreased job satisfaction, increased medical errors, and increased attrition, which incurs a financial expense for the employing agency.

\section{Discussion}

Using the hybrid approach to concept analysis, this study attempted to clarify and determine the meaning of a successfully transitioned NLRN through a synthesis of the related literature and the in-depth explorations of registered nurses' perceptions. The findings of the fieldwork phase were highly similar to those of the theoretical phase. Accordingly, the definition of the successfully transitioned NLRN is proposed as follows:

"Successfully transitioned NLRNs demonstrate a functional proficiency in assessment, communication, time management, advocacy, prioritization, clinical decision making, and care coordination. They are able to apply knowledge and adapt appropriately in new clinical situations and are assertive in accessing resources to ensure patient safety, enhance their performance and improve their skill set."

In the nursing literature, the successfully transitioned NLRN is not clearly defined and developed as a concept. The lack of common perception of what it means to successfully transition creates difficulty in identifying the boundaries of the concept and promotes varying expectations within the profession and across disciplines. An integrative review by Rush et al. [40]. identified a deficit in clarity regarding the concept of "transition" in nursing. Common surrogate terms found in the literature included "job readiness," "practice readiness," and "readiness for practice," while related terms included the "transition to practice" and "entry into the workplace" [34].

The literature suggested a NLRN who has transitioned in their practice demonstrates readiness for practice or is practiceready. However, that concept remains vague and ambiguous and lacks specificity [26]. Developing a shared understanding of transitional success will provide a foundation for building greater collaboration in the preparation, transition, and so- 
Brown et al, Journal of Nursing 2021,

cialization of NLRNs into the nursing workforce. Therefore, we sought to analyze the concept and determine a definition and identification of its attributes.

Through our exploration of the literature and the nurse participants' perspectives, we have determined completion of a prescribed period of orientation and participation in a structured transitional support program does not equate to a successful transition to practice for the NLRN. Participants from both groups agreed that it takes a NLRN 12 to 18 months to achieve the established attributes, supporting the findings of Oermann et al. [10].

The findings suggest that a successful transition implies that the NLRNs are competent in skill acquisition and possess some personal and professional attributes that support their ability to communicate, collaborate, prioritize, delegate, make clinical judgments, and support their professional growth independently [37]. These attributes contribute to the development of confidence in their performance and competence in their practice but also promote patient safety in a complex and changing healthcare environment. One of the main consequences of a successful transition identified in this study is competence in clinical decision-making and critical thinking. This finding supports findings from Theisen \& Sandou; [35] NLRNs who provide safe care are ones who can think critically and make sound clinical decisions, a hardship encountered by inexperienced NLRNs.

A successful transition to practice mirrors the evolution in Benner's novice to expert theory as the NLRN progresses through a process of personal and professional development from being a novice in their role to being an exper [31]. In addition, Duchscher $[7,30]$ posits that the NLRN who has successfully transitioned has developed in the areas of doing, knowing, and thinking to ensure safe care.

This study also adds that though transitional success was used interchangeably with practice readiness, readiness for practice is contextual and depends on the nurse's preparedness to be able to begin practice in a given clinical setting [8]. According to Oermann et al. [10] readiness implied a confidence in clinical skill performance and adequate pre-licensure clinical experiences. In comparison, transitional success is more related to the nurse's ability to provide safe basic care and fulfill the standards of practice independently, regardless of the setting.

In addition, the achievement of transitional success is highly individualized and is influenced by multiple variables. Transitional success is individualized and shaped by the context of the clinical environment and the various units or specialties in which the NLRN is working, and their own personal experiences, as some NLRNs may encounter patients and patient acuities they were never exposed to in academia.

What is vital in facilitating a successful transition is that the NLRN is supported and provided with the support necessary to complete the transition period successfully. This is pivotal in determining if the NLRN can safely integrate into the workforce as an independent practitioner.
Based on this study's findings and the wide variability in the time frame to achieve a competent level of performance, a shift in the current practice environment's culture is required for a successful transition of the NLRN into practice. We suggest there is scope for more focus on how transitional success is measured and how to support the individual needs of the NLRN to achieve a successful transition, rather than a prescribed time frame for transition.

Despite literature exploring practice readiness, the practice preparation gap, and the difficulties encountered by the NLRN, to the best of our knowledge, this is the first study to explore the conceptual meaning of a transitional success for the NLRN. The current research provides theoretical and empirical evidence regarding the concept's attributes and consequences and provides a conceptual definition based on these findings. These results also serve as a catalyst for further research to develop a psychometrically validated tool to measure its attributes.

\section{Limitations}

This study was conducted in the northern United States and may not be transferrable to all geographic settings. The small sample size $(n=17)$ might be a possible limitation of the study. However, organizations within multiple states were represented, and the sample size was determined by data saturation when interviews failed to return new insight. A small sample size might affect generalizability but not transferability. The nurses in a non-leadership role outnumbered the administrators, resulting in an uneven representation of both groups. Data saturation was achieved despite this discrepancy, as both groups identified similar attributes, and no new data was uncovered.

\section{Recommendations for Future Directions}

The literature search failed to identify a definition of a NLRN successfully transitioned in their practice or identify a single tool that explicitly measures NLRN transitional success. Based on this deficit, we have presented a conceptual definition. Recommendations for future research include developing a psychometrically validated survey instrument to measure the attributes of the successfully transitioned NLRN and operationalize this definition.

\section{Implications to Nursing Practice}

A successful transition to independent clinical practice is crucial for patient safety and positive healthcare outcomes. The literature identifies the difficulties NLRNs experience in socializing and transitioning into their roles, often resulting in the NLRN leaving the profession prematurely based on their experiences [11-16]. This research highlights the importance of ensuring a successful transition and the need to develop a more rigorous definition of the concept. A definition provides a consistent benchmark to measure transitional progress and successful transition to independent practice for the NLRN.

The transitional progress of the NLRN requires consistent and continuous evaluation to gauge readiness for practice 
and ensure safety in engaging in independent practice. More research is needed to develop a survey instrument to measure the attributes of the successfully transitioned nurse. This new survey instrument may be able to assess effective transition and preparation for independent practice and evaluate the level of support required to achieve this goal. The information gained from using this tool could be used to establish more robust and deliberate transitional support outside the orientation period, focusing on the professional development and refinement of the individual NLRN transitioning to independent practice.

\section{Conclusion}

This paper's purposes were to (1) analyze the concept of transitional success and (2) develop a conceptual definition of the successfully transitioned NLRN. Clearly defined concepts are foundational for establishing theories and frameworks for use in nursing and clinical practice related to NLRN performance. We identified that despite the perceived deficiencies and challenges experienced by the NLRN and the need for a successful transition, the concept itself remains ill-defined. Based on our findings, transitional success should not be determined by a linear model of skill acquisition or time but to also consider context and the individual characteristics of the NLRN.

As patient safety and nurse job satisfaction hinge on the successful transition and socialization of the NLRN into their roles and practice, it is essential to define the successfully transitioned nurse's attributes. The concept lacked a clear and precise definition though the implications have been widely discussed in the literature. This lack of conceptual clarity can lead to deficits in how the NLRN is viewed in terms of readiness for independent practice. Built on this discovery and through applying the hybrid model, we proposed a definition addressing specific knowledge, skills, and attitudes inherent in a successfully transitioned NLRN. With the concept now more clearly defined, stakeholders involved in the transitional process can more purposefully and critically evaluate the NLRN in terms of their individual needs, readiness for independent practice, and socialization into their role before withdrawing transitional support entirely. Having a conceptual definition of the successfully transitioned NLRN is a prerequisite for more research, specifically the development of a survey instrument to measure the operational concepts.

\section{Competing interests}

The authors declare that they have no competing interests.

Authors' contributions

\begin{tabular}{|l|l|l|}
\hline Authors' contributions & JB & JN \\
\hline Research concept and design & & \\
\hline Collection and/or assembly of data & & \\
\hline Data analysis and interpretation & & \\
\hline Writing the article & & \\
\hline Critical revision of the article & & \\
\hline Final approval of article & & \\
\hline
\end{tabular}

\section{Acknowledgements}

This research was supported (in part) by a grant from the Center for Research, College of Science and Health, William Paterson University of New Jersey.

\section{Publication history}

Editor: David Reiss, Imperial College London, UK.

Received: 09-Sept-2021 Final Revised: 15-Oct-2021

Accepted: 20-Oct-2021 Published:

\section{References}

1. Bennett, L., Grimsley, A., Grimsley, L., \& Rodd, J. (2017). The gap between nursing educationand clinical skills. ABNF Journal, 28(4), 96-102. Retrieved from https://ezproxy.wpunj.edu/login?url=https:// searchproquestcom.ezproxy.wpunj.edu/docview/2039834640?account $\mathrm{id}=15101$

2. Hoffart, N., Waddell, A., \& Young, M.B. (2011). A model of new nurse transition. Journal ofProfessional Nursing: Official Journal of the American Association of Colleges of Nursing, 27(6), 334-43.

3. Murray, M., Sundin, D., \& Cope, V. (2019). Benner's model and Duchscher's theory: Providing the framework for understanding new graduate nurses' transition to practice.Nurse Education in Practice, 34 199-203. https://doi.org/10.1016/j.nepr.2018.12.003

4. Thomas, C. M., Bertram, E., \& Allen, R. L. (2012). The transition from student to new registerednurse in professional practice. Journal for Nurses in Professional Development, 28(5), 243-249.

5. Kramer, M. (1975). Reality shock: Why nurses leave nursing. AJN The American Journal of Nursing, 75(5), 891.

6. Valdez, A. (2008). Transitioning from Novice to Competent: What Can We Learn From theLiterature About Graduate Nurses in the Emergency Setting? Journal of EmergencyNursing, 34(5), 435-440. https://doi. org/10.1016/j.jen.2007.07.008

7. Duchscher, J. (2008). A process of becoming: the stages of new nursing graduate professionalrole transition. The Journal of Continuing Education in Nursing, 39(10), 441-450. https://doi.org/10.3928/0022012420081001-03

8. Wolff, A., Pesut, B., \& Regan, S. (2010). New graduate nurse practice readiness: Perspectives onthe context shaping our understanding and expectations. Nurse Education Today, 30(2), 187-191. https://doi. org/10.1016/j.nedt.2009.07.011

9. Hofler, L., \& Thomas, K. (2016). Transition of New Graduate Nurses to the Workforce: Challenges and Solutions in the Changing Health Care Environment. North CarolinaMedical Journal (Durham, N.C.), 77(2), 133-136. https://doi.org/10.18043/ncm.77.2.133

10. Oermann, M. H., Poole-Dawkins, K., Alvarez, M. T., Foster, B. B., \& O'Sullivan, R. (2010). Managers' perspectives of new graduates of accelerated nursing programs: How do theycompare with other graduates? The Journal of Continuing Education in Nursing, 41(9), 394400

11. Duclos-Miller, P.A. (2011). Successful graduate nurse transition: Meeting the challenge. Nurseleader 9(4), 32,49-35,49. doi:10.1016/j. $\mathrm{mnl}$.2011.05.006

12. Hickerson, K.A., Taylor, L.A., \& Terhaar, M. (2016). The PreparationPractice gap: An integrative literature review. Journal of Continuing Education in Nursing, 47(1), 17-23.

13. Laschinger, H., Cummings, G., Leiter, M., Wong, C., MacPhee, M., Ritchie, J., Wolff, A., Regan, S., Rhéaume-Brüning, A., Jeffs, L., Young-Ritchie, C., Grinspun, D., Gurnham, M., Foster, B., Huckstep, S., Ruffolo, M., Shamian, J., Burkoski, V., Wood, K., \& Read, E. (2016). Starting Out: A time-lagged study of new graduate nurses' transition topractice. International Journal of Nursing Studies, 57, 82-95. https://doi.org/10.1016/j. ijnurstu.2016.01.005

14. van Rooyen, D., Jordan, P., ten Ham-Baloyi, W., \& Caka, E. (2018). A comprehensive literature review of guidelines facilitating transition of newly graduated nurses toprofessional nurses. Nurse Education in Practice, 30, 35-41. https://doi.org/10.1016/j.nepr.2018.02.010 
Brown et al, Journal of Nursing 2021,

15. McCalla-Graham, J. A., \& De Gagne, J. C. (2015). The Lived Experience of New GraduateNurses Working in an Acute Care Setting. The Journal of Continuing Education in Nursing, 46(3), 122-128. https://doi. org/10.3928/00220124-20150220-17

16. Blevins, S., \& Millen, E. (2016). Foundation for New Graduate Nurse Success. MedsurgNursing, 25(3), 194-201.

17. Goode, C., Reid Ponte, P., \& Sullivan Havens, D. (2016). Residency for Transition into Practice:An Essential Requirement for New Graduates from Basic RN Programs. Journal of Nursing Administration, 46(2), 82-86. https://doi.org/10.1097/NNA.0000000000000300Letourneau, R., \& McCurry, M. (2019). The Effect of Transition to Practice Programs on the

18. Self-Assessment of Newly Licensed Registered Nurses' Confidence in Quality and SafetyCompetency Attainment. Nursing Education Perspectives, 40(3), 151-156. https://doi.org/10.1097/01. NEP.0000000000000438

19. Clark, C., \& Springer, P. (2012). Nurse residents' first-hand accounts on transition to practice. Nursing Outlook, 60(4), e2-e8. https://doi. org/10.1016/j.outlook.2011.08.003

20. Schwartz-Barcott, D. \& Kim, S. (2000). An expansion and elaboration of the hybrid model of concept development. In B. L. Rodgers \& K. A. Knafl (Eds.), Concept development in nursing: Foundation, techniques and applications (2nd ed.). W.B. Sanders, Philadelphia.

21. Knafl, K. A., \& Rodgers, B. L. (1993). Concept development in nursing foundations,techniques, and applications. Philadelphia, PA, W.B. Saunders Company Creswell, J. W. (2007). Qualitative inquiry and research design: Choosing among fiveapproaches (2nd ed.). Thousand Oaks, CA: SAGE. Creswell, J. W. (2012). Educational research: Planning, conducting, and evaluating quantitativeand qualitative research. Boston, MA: Pearson. Creswell, J. W. (2014). Research design: Qualitative, quantitative and mixed methodsapproaches (4th ed.). Thousand Oaks, CA: SAGE.

22. Romyn, D., Linton, N., Giblin, C., Hendrickson, B., Houger Limacher, L., Murray, C., Nordstrom, P., Thauberger, G., Vosburgh, D., Vye-Rogers, L., Weidner, A., \& Zimmel, C. (2009). Successful Transition of the New Graduate Nurse. International Journal ofNursing Education Scholarship, 6(1), 34-Article 34. https://doi.org/10.2202/1548923X.1802

23. Wolff, A., Regan, S., Pesut, B., \& Black, J. (2010). Ready for What? An Exploration of theMeaning of New Graduate Nurses' Readiness for Practice. International Journal of Nursing Education Scholarship, 7(1), 7Article7. https://doi.org/10.2202/1548923X.1827

24. Brewer, C. S., Kovner, C. T., Greene, W., Tukov-Shuser, M., \& Djukic, M. (2012). Predictors of actual turnover in a national sample of newly licensed registered nurses employed inhospitals. Journal of advanced nursing, 68(3), 521-538. https://doi.org/10.1111/ j.13652648.2011.05753.x

25. Merriam-Webster. (n.d.). Transition. In Merriam-Webster.com dictionary. Retrieved March 17, 2021, from https://www.merriam-webster.com/ dictionary/transition

26. Phillips, C., Kenny, A., Esterman, A., \& Smith, C. (2014). A secondary data analysis examining the needs of graduate nurses in their transition to a new role. Nurse education in practice,14(2), 106-111. https://doi. org/10.1016/j.nepr.2013.07.007

27. Duchscher J.E.B. (2009). Transition shock: The initial stage of role adaptation for newlygraduated registered nurses. Journal of Advanced Nursing, 65, 1103 - 1113. 10.1111/j.1365-2648.2008.04898.x

28. Benner, P. (1984). From novice to expert. Menlo Park, 465-468.

29. El Haddad, M., Moxham, L., \& Broadbent, M. (2017). Graduate nurse practice readiness: Aconceptual understanding of an age-old debate. Collegian, 24(4), 391-396.

30. Wiersma, G., Pintz, C., \& Fraser Wyche, K. (2020). Transition to Practice Experiences of New Graduate Nurses from an Accelerated Bachelor of Science in Nursing Program: Implications for Academic and Clinical Partners. The Journal of Continuing Education inNursing, 51(9), 433-440. https://doi.org/10.3928/00220124-20200812-09

31. Mirza N., Manankil-Rankin L., Prentice D., Hagerman L., Draenos C. (2019) Practice readinessof new nursing graduates: A concept analysis. Nurse Education in Practice, 37, 68-74. 10.1016/j.nepr.2019.04.009
32. Theisen, J.L. \& Sandau, K.E. (2013). Competency of New Graduate Nurses: A Review of Their Weaknesses and Strategies for Success. The Journal of Continuing Education inNursing, 44(9), 406-414. https://doi. org/10.3928/00220124-20130617-38

33. Sheldon, L. K., \& Hilaire, D. M. (2015). Development of communication skills in healthcare:Perspectives of new graduates of undergraduate nursing education. Journal of NursingEducation and Practice, 5(7), 30

34. Sparacino, L. L. (2016). Faculty's Role in Assisting New Graduate Nurses' Adjustment toPractice. SAGE Open Nursing, http://dx.doi.org.ezproxy. wpunj.edu/10.1177/2377960816635182

35. Brown, J. E. (2019). Graduate Nurses' Perception of the Effect of Simulation on Reducing theTheory-Practice Gap. SAGE Open Nursing, 5, 2377960819896963.

36. DeGrande, H., Liu, F., Greene, P., \& Stankus, J. (2018). The experiences of new graduate nurses hired and retained in adult intensive care units. Intensive \& Critical Care Nursing,49, 72-78. https://doi.org/10.1016/j. iccn.2018.08.005

37. Washington, G. (2012). Performance Anxiety in New Graduate Nurses: Is It for Real? Dimensions of Critical Care Nursing, 31(5), 295-300.https:// doi.org/10.1097/DCC.0b013e3182619b4c

38. Rush, K., Adamack, M., Gordon, J., Lilly, M., \& Janke, R. (2013). Best practices of formal newgraduate nurse transition programs: An integrative review. International Journal of Nursing Studies, 50(3), 345-356. https://doi.org/10.1016/j.ijnurstu.2012.06.009

39. Clipper, B., \& Cherry, B. (2015). From Transition Shock to Competent Practice: DevelopingPreceptors to Support New Nurse Transition. The Journal of Continuing Education inNursing, 46(10), 448-454. http://dx.doi. org.ezproxy.wpunj.edu/10.3928/0022012420150918-02

40. Benner, P. (2001). From novice to expert: excellence and power in clinical nursing practice (Commemorative ed.), Prentice-Hall, Upper Saddle River (NJ) (2001) - found in Valdez,2008 - Transitioning from Novice to Competent: What Can We Learn from the LiteratureAbout Graduate Nurses in the Emergency Setting?

41. Moher D, Liberati A, Tetzlaff J, Altman DG, The PRISMA Group (2009). Preferred Reportingltems for Systematic Reviews and Meta-Analyses: The PRISMA Statement. BMJ 2009;339:b2535, doi: 10.1136/bmj.b2535

Citation:

Brown J and Nocella J. Developing a Conceptual Definition of the Successfully Transitioned Newly Licensed Registered Nurse (NLRN). J Nurs. 2021; 8:1. http://dx.doi.org/10.7243/2056-9157-8-1 